\title{
The development of transient fingering patterns during the spreading of surfactant coated films
}

\author{
Omar K. Matar \\ Department of Chemical Engineering and Chemical Technology, Imperial College, London, \\ England SW7 2BY \\ Sandra M. Troian ${ }^{\text {a) }}$ \\ Department of Chemical Engineering, Princeton University, Princeton, New Jersey 08544-5263
}

(Received 6 June 1998; accepted 19 July 1999)

\begin{abstract}
The spontaneous spreading of an insoluble surfactant monolayer on a thin liquid film produces a complex waveform whose time variant shape is strongly influenced by the surface shear stress. This Marangoni stress produces a shocklike front at the leading edge of the spreading monolayer and significant film thinning near the source. For sufficiently thin films or large initial shear stress, digitated structures appear in the wake of the advancing monolayer. These structures funnel the oncoming flow into small arteries that continuously tip-split to produce spectacular dendritic shapes. A previous quasisteady modal analysis has predicted stable flow at asymptotically long times [Phys. Fluids A 9, 3645 (1997)]. A more recent transient analysis has revealed large amplification in the disturbance film thickness at early times [O. K. Matar and S. M. Troian, "Growth of nonmodal transient structures during the spreading of surfactant coated films," Phys. Fluids A 10, 1234 (1998)]. In this paper, we report results of an extended sensitivity analysis which probes two aspects of the flow: the time variant character of the base state and the non-normal character of the disturbance operators. The analysis clearly identifies Marangoni forces as the main source of digitation for both small and large wave number disturbances. Furthermore, initial conditions which increase the initial shear stress or which steepen the shape of the advancing front produce a larger transient response and deeper corrugations in the film. Disturbances applied just ahead of the deposited monolayer rapidly fall behind the advancing front eventually settling in the upstream region where their mobility is hampered. Recent findings confirm that additional forces which promote film thinning can further intensify disturbances [O. K. Matar and S. M. Troian, "Spreading of surfactant monolayer on a thin liquid film: Onset and evolution of digitated structures," Chaos 9, 141 (1999). The transient analysis presented here corroborates our previous results for asymptotic stability but reveals a source for digitation at early times. The energy decomposition lends useful insight into the actual mechanisms preventing efficacious distribution of surfactant. (C) 1999 American Institute of Physics. [S1070-6631(99)01111-3]
\end{abstract}

\section{INTRODUCTION}

Surfactant molecules play a vital role in numerous household, industrial, and biological processes. Their ability to lower surface tension significantly improves the wetting and spreading capability of commonplace substances like shampoo, detergent, ink, paint, herbicide and medicine. Surfactants produced naturally in mammalian systems are especially important in maintaining lung compliance by reducing the surface tension of the liquid film which coats the interior of pulmonary airways. Deficiencies can produce pulmonary edema or other serious respiratory difficulties. ${ }^{1}$ Premature infants, for example, often suffer from an insufficient supply of lung surfactant which can suddenly lead to respiratory distress syndrome. This fatal condition can easily be relieved by the inhalation of a suspension of animal or synthetic lung surfactant. This quick and simple technique has achieved good clinical success ${ }^{2}$ although further improvements de-

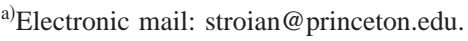

pend on a better understanding of the surfactant transport from the upper to the lower recesses of the lung.

The transport of exogenous lung surfactant along the alveolar lining is a complicated process involving the rapid distribution of a multicomponent surfactant formulation to a liquid layer with nonuniform surface properties. The principle mechanism for rapid distribution of surfactant, however, can be modeled as the spontaneous spreading of a surfactant monolayer along a liquid layer of higher surface tension. ${ }^{3-5}$ At the junction where the initial monolayer joins the native liquid film, there exists a shear stress whose magnitude is directly proportional to the difference in surface tension across the boundary. This shear stress pulls liquid and surfactant towards regions of higher surface tension. From the point of view of minimizing the free energy associated with the spreading process, the regions of higher surface tension are rapidly coated with surfactant thereby reducing the surface energy of the entire liquid layer. In biological or industrial applications, the effectiveness of the coating process is improved if the spreading is rapid and uniform, if 
the liquid layer is not ruptured by large surface stresses, and if the liquid flow remains stable and continuous until complete coverage is attained.

Simple model experiments during the past several years have shown that the spreading of surfactant films on a thin liquid layer of higher surface tension is neither a uniform nor stable process ${ }^{6-12}$ Often the spreading monolayer produces significant film thinning near the surfactant source. This region is then observed to undergo transverse corrugations which funnel the oncoming flow into small arteries that continuously branch and tip-split. These patterns, which develop on the backside of the surfactant leading edge, resemble the shape of fingering patterns in viscous fingering. ${ }^{13}$ Unlike the viscous fingering problem, however, the surfactant patterns do not derive from the higher viscosity of the displaced fluid. Typical patterns which develop during the spreading of surfactant on a thin water film are shown in Fig. 1. These digitated structures have been observed in many different experiments. They include studies with soluble or insoluble surfactant (with ringed or branched molecular structures); solutions which are both above and below the critical micelle concentration; surfactants with head group charge that is neutral, zwitterionic or of opposite charge to the wetted substrate; and surfactants spreading on film thicknesses ranging from fractions of a micron to millimeters. The fingering behavior has also been documented in both rectilinear and axisymmetric geometry, for delivery from a finite or infinite source, and for geometries in which gravity either enhances or retards the flow. The fact that the digitated patterns never appear when spreading a solution directly onto a dry substrate indicates that Marangoni stresses are in some way responsible for the unusual spreading behavior. The question of whether these patterns are long lived or just transient structures has not yet been answered experimentally. In most cases, the ambient atmosphere is not controlled and evaporation disrupts the spreading process, especially in the thinnest portions of the liquid film. In addition, adjacent fingers can coalesce at long times.

Because of the ubiquity and importance of the process whereby surfactant molecules are transported along the surface of a thin liquid layer, theoretical efforts have concentrated on developing a set of coupled equations which can accurately describe the spreading behavior. By appealing to the lubrication approximation, which assumes that the film thickness is much smaller than the extent of spreading, and by adopting a linear equation of state which relates the surface tension to the local concentration of surfactant, a pair of couple non-linear equations have been derived describing the spatiotemporal evolution of the liquid film thickness, $h(\mathbf{x}, t)$, and surface surfactant concentration, $\Gamma(\mathbf{x}, t)$. Over the years, these equations have been extended $d^{4,5,14-16}$ to include not only the Marangoni shear stress, but capillary effects arising from the surface deformation, bulk and surface diffusion of surfactant, gravitational terms which flatten the shocklike rim at the leading edge, and disjoining forces which either promote or retard film thinning. The resulting fourth-order equations have been solved numerically subject to realistic initial and boundary conditions. These numerical studies have shown that a thin liquid film contacted by a surfactant

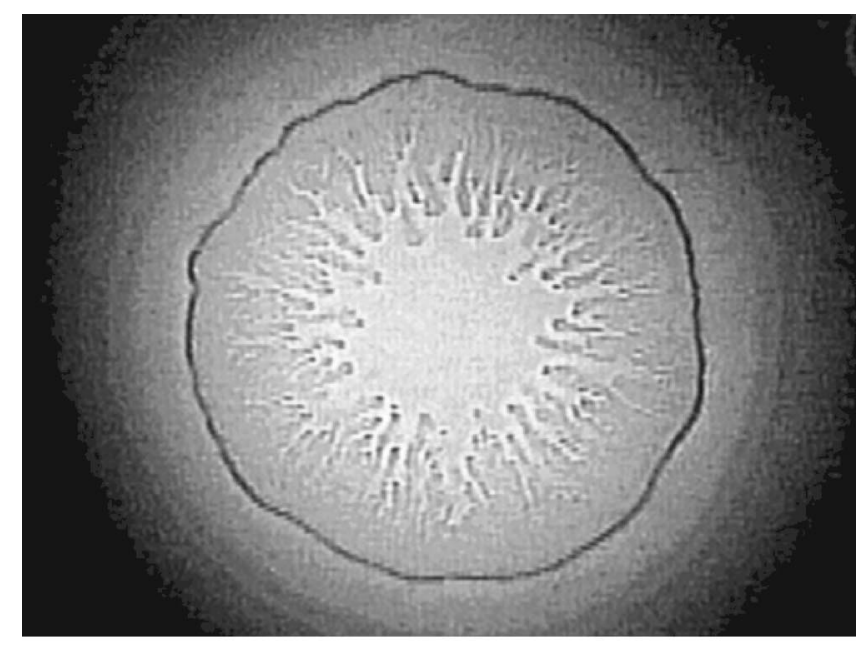

(a)

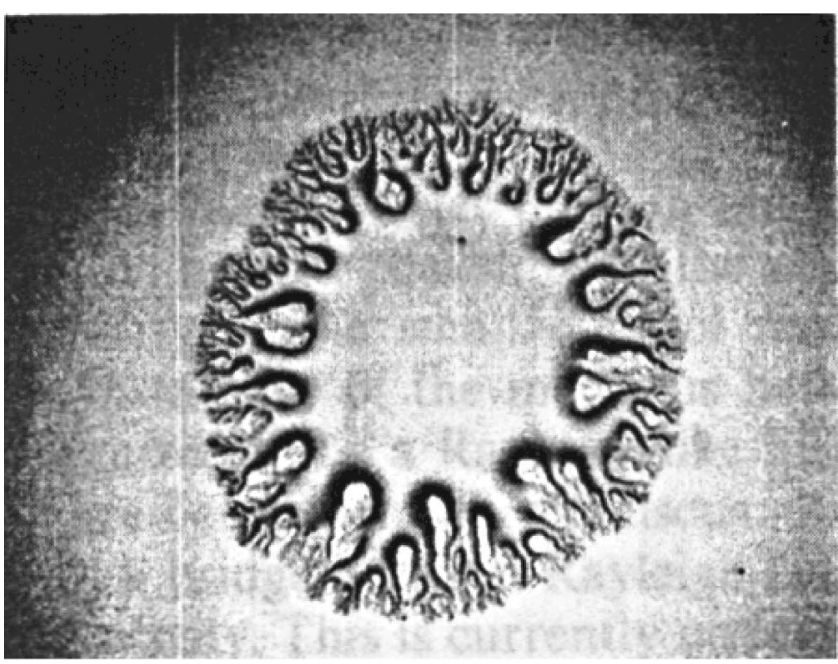

(b)

FIG. 1. Surface patterns observed during the spreading of a surfactant droplet on a thin water film. (a) $15 \mu \ell$ drop of $6.6 \mathrm{mM}$ aqueous SDS solution spreading on water film of thickness $H_{o} \approx 1 \mu, 3 \mathrm{~s}$ after deposition (Ref. 8). Black outer ring, of diameter $9.6 \mathrm{~cm}$., demarcates the leading edge of the surfactant front. (b) A microdroplet of $\mathrm{C}_{12} \mathrm{E}_{10}$ in ethylene glycol spreading on a water layer estimated to be $10^{-2} \mu$ in thickness (Ref. 12). Because the initial water film is ultra thin, the surfactant leading edge is no longer visible.

monolayer is spontaneously pulled in the direction of increasing surface tension. The resulting stress profile produces a thickened advancing rim and strong film thinning near the deposition region. Key flow variables like the speed of propagation, the time variant shape of the spreading film, and the spatial distribution of surfactant along the surface, strongly depend on the magnitude of the initial shear stress and film thickness, as well as the viscosity of the liquid support and the surfactant equation of state.

A straightforward modal analysis of the coupled equations governing the response of $h$ and $\Gamma$ to small disturbances is complicated by two very important aspects of the flow; (i) the base state profiles for $h$ and $\Gamma$ are time variant due to the decreasing shear stress (since a finite mass of surfactant is distributed over an ever larger area) and (ii) their spatial dependence produces non-normal disturbance 
operators. The first aspect introduces the complication that any conclusion inferred about the "stability" of the system to small disturbances is only meaningful with respect to the temporal behavior of the base state. There are many fluid dynamical systems which exhibit this difficulty and Shen's original measure for investigating the "momentary stability" of time variant base states proves a suitable framework for our system. ${ }^{17,18}$ The second aspect involving nonnormality stems from the fact that even if the base state is frozen in time, the free surface shape and concentration distribution are spatially dependent. It is well known that in such cases a modal type analysis will only reveal the asymptotic behavior of the system as $t \rightarrow \infty .{ }^{19,20}$ This property implies that even if the largest real part of the eigenspectrum is negative, the system may still harbor large disturbance amplification at early times. The analysis in the later sections attempts to separate the time variance from the nonnormal property by studying the stability of disturbances about a frozen base state.

What simplifications can be performed to eliminate the time variance of a base state? For a finite monolayer whose spreading is only controlled by Marangoni forces, there exist no steady-state solutions. One can derive self-similar solutions to the spreading process in a stretched coordinate whose length is coincident with the monolayer leading edge. The addition of other forces, like capillarity, surface diffusion, gravity, or van der Waals precludes even self-similar solutions. Numerical studies have shown, however, that if these additional forces are weaker than the Marangoni force, then after the impact of initial conditions has died away, the film thickness and concentration profiles approach a selfsimilar form dominated by the surface shear stress. In this case, both profiles assume a simple linear form. One can therefore freeze the base states at their (long time) selfsimilar form and perform a linear stability analysis on these shapes.

This approach has been implemented in the literature in two successive steps. The first theoretical attempt at revealing the mechanism leading to fingered spreading relied on the remarkable similarity between the surfactant patterns and those produced by miscible or immiscible viscous fingering. ${ }^{13}$ Within a long wavelength approximation, the variation in film thickness near the source was neglected and only disturbances in the surfactant concentration allowed. The concentration base state was then frozen about its selfsimilar form. This approximation immediately led to a simplified time dependent Laplacian equation for the disturbance concentration with positive growth rate for small wave numbers. In mapping the gradient in surfactant concentration onto the gradient in the applied pressure, the mathematical analogy to the viscous fingering problem was complete.

This analysis suggested that the fingering behavior observed in surfactant spreading problems derived from a Laplacian driven process as occurs in viscous fingering or diffusion-limited aggregation, ${ }^{21}$ even though its physical origin was different. This argument states that if a velocity field is directly proportional to the driving force (be it a gradient in applied pressure, surfactant concentration or electric potential) and if the local driving force increases upon advanc- ing the interface into a region where there exists a constant gradient in the relevant field variable, then infinitesimal protrusions will advance faster than neighboring portions of the front and destabilize the interface. In fluid systems, the speed of the advancing front is controlled by the local pressure gradient as well as the local mobility. In viscous fingering, for example, the displaced fluid is of higher viscosity and lower mobility than the penetrating fluid. In the surfactant problem, the initial shear stress strongly thins the area just ahead of the surfactant reservoir producing a region of decreased mobility. Just as the source of instability in the viscous fingering case has been traced to a region of adverse mobility, so too was the thinned region responsible for the dendritic patterns in the surfactant spreading problem. Despite the universal appeal of this analogy, a more rigorous stability analysis was required which allowed disturbance variations in both the film thickness and surfactant concentration. Results from quasisteady calculations using selfconsistent, self-similar solutions for the base state but allowing disturbances in both variables ${ }^{22,23}$ proved that the spreading dynamics was stable to disturbances of all wave numbers. The inclusion of additional but weaker forces like capillarity and surface diffusion confirmed linearly stable flow. ${ }^{24,25}$

These quasisteady calculations highlighted certain important features of the spreading process. First, the analyses underscored the importance of allowing variations in both the film thickness and surfactant concentration. Allowing self-consistent disturbances in both field variables produced a cooperative stabilizing response. Second, the null results obtained raised the obvious possibility that the fingering process might be an early time response which was obscured by assuming base state solutions of (late time) self-similar form. The experimental evidence in the literature ${ }^{6,7,9-12}$ suggests that the onset for the fingering process is very rapid, appearing almost immediately behind the spreading front. Although the onset time has not been studied systematically, the time scale for the appearance of surface corrugations is on the order of a shear time, $\tau \sim L_{0} / U_{0}$, where $L_{0}$ is the initial extent of the monolayer and $U_{0}$ the initial spreading speed. This information, though not definitive, suggests that the spreading behavior at early times (when the shear stress is largest) may differ in response from the late time dynamics. Investigating the early time dynamics for a base state which is time variant, however, requires a more general measure of the growth or decay of disturbances since the underlying reference state is changing in time as well.

Finally, there is another critical issue raised by the modal analyses performed in the past which requires discussion. Even when neglecting the time variance of the base states, the self-similar solutions possess spatial inhomogeneity; namely, the film thickness resembles an increasing linear ramp from the source to the leading edge while the surfactant concentration resembles a decreasing linear ramp. ${ }^{5,16,23}$ As described in more detail in later sections, this spatial dependence directly introduces non-normality of the disturbance operators. A straightforward modal analysis, whereby the eigenvalues of the disturbance operators are computed directly, can only determine the stability of the system as time 
$t \rightarrow \infty$. Therefore, the results obtained by the previous quasisteady calculations may just have signaled that the spreading process is stable to disturbances of all wave numbers at late times. Information about the early time behavior was inaccessible through these computations.

The majority of this paper, therefore, deals with disturbances in the film thickness and surfactant concentration applied at very early times. For comparison, we include some results of the effect of applying disturbances at intermediate times only after the base state solutions have begun settling down to a similar form. Both the early and intermediate time calculations clearly show that this system harbors the potential for large transient growth. The amplification of disturbances eventually decays away at late times when the driving force for spreading has weakened considerably.

In summary, the linearized transient description strongly suggests that a surfactant monolayer spreading on a thin viscous film initially exhibits extreme sensitivity to infinitesimal transverse disturbances in the film thickness or surfactant concentration. These disturbances are amplified on time scales comparable to a Marangoni shear time. In accordance with Shen's framework for investigating the "momentary stability" of time variant base states, ${ }^{17,18}$ we propose a quantitative measure for the amplitude and rate of disturbance growth. This information is then used to identify the physical mechanisms responsible for the onset of liquid channeling and fingering. The long time limit of our transient calculations corroborate our earlier findings of asymptotic stability using the quasisteady approach. The calculations presented here, however, establish a timeline for understanding the spatial and temporal response of the film thickness and surfactant concentration to initial disturbances and their evolution toward asymptotically stable states. Although beyond the scope of this paper, we have also carried out calculations to determine how important might be nonlinear effects as the source of the digitation. The interested reader is referred to Ref. 24 for a discussion of this issue.

\section{PROBLEM FORMULATION}

\section{A. Governing equations}

Consider a quiescent Newtonian liquid layer of initial uniform thickness $H_{0}^{*}$, viscosity $\mu^{*}$, and density $\rho^{*}$, resting on a flat solid substrate, as shown in Fig. 2. The liquid film is contacted by a monolayer of insoluble surfactant of initial extent $L_{0}^{*}$ where $\epsilon=H_{0}^{*} / L_{0}^{*} \ll 1$ in accordance with the lubrication approximation. Because of the mismatch in surface tension at the junction between clean and contaminated surface, there spontaneously develops a large shear stress which drives the liquid film toward regions of higher surface tension. The spontaneous spreading reflects the balance between the surface shear stress of order $\Pi * / L_{0}^{*}$ and the viscous drag of order $\mu^{*} U^{*} / H_{0}^{*}$. The parameter $\Pi^{*}=\sigma_{0}^{*}-\sigma_{m}^{*}$ denotes the maximal spreading pressure where $\sigma_{0}^{*}$ is the surface tension of the clean liquid layer and $\sigma_{m}^{*}$ the initial surface tension of the coated liquid layer. The characteristic spreading velocity, $U^{*}$, is determined from a force balance to be $U^{*}$ $=\varepsilon \Pi * / \mu^{*}$. This velocity is sometimes termed the Marangoni spreading velocity because of its physical origin.

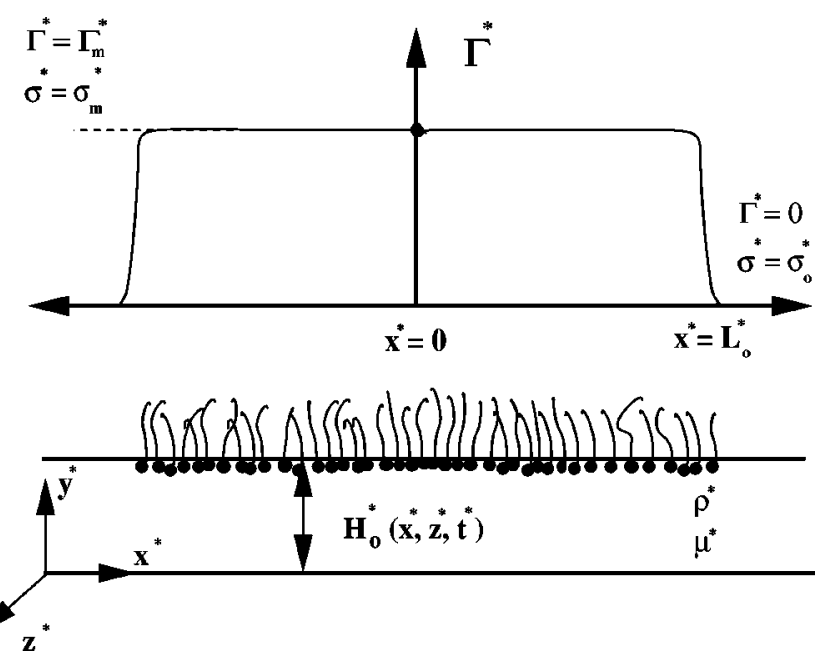

FIG. 2. Schematic diagram of a surfactant monolayer about to spread along the surface of a thin liquid film.

The equations of motion are nondimensionalized by the horizontal scale, $L_{0}^{*}$, the vertical scale, $H_{0}^{*}$, the horizontal velocity, $U^{*}$, and the vertical velocity, $\varepsilon U^{*}$. The characteristic shear time for the spreading process is $\mu^{*} L_{0}^{*} / \varepsilon \Pi^{*}$ while the characteristic pressure in the film is given by $\Pi^{*} / H_{0}^{*}$. The dimensionless spreading pressure is defined to be $\left(\sigma^{*}\right.$ $\left.-\sigma_{m}^{*}\right) / \Pi^{*}$, which defines the ratio of the local driving force to the maximum driving force for spreading. In this paper we consider spreading dominated by Marangoni stress in the presence of additional weaker forces like capillarity and surface diffusion. Other forces like gravity, disjoining pressure or diffusion from the bulk to the interface can be easily incorporated.

We define a rectilinear spreading geometry such that $x^{*}$ denotes the horizontal direction, $y^{*}$ the vertical direction, and $z^{*}$ the transverse direction. The spreading process occurs in the $x^{*}-z^{*}$ plane and $y^{*}=0$ locates the vertical position of the solid support. With the choice of scalings described, the dimensionless equations for incompressibility and momentum conservation become

$$
\begin{aligned}
& u_{x}+\mathrm{v}_{y}+w_{z}=0, \\
& 0=-p_{x}+u_{y y}+O\left(\varepsilon^{2}\right), \\
& 0=-p_{y}+O\left(\varepsilon^{2}\right), \\
& 0=-p_{z}+w_{y y}+O\left(\varepsilon^{2}\right),
\end{aligned}
$$

wherein the horizontal, vertical, and transverse velocity fields are represented by $u, \mathrm{v}$, and $w$, respectively. The subscripts denote partial differentiation with respect to $x, y$, or $z$. The boundary conditions used to solve Eqs. (1)-(4) include impenetrability and no slip at the solid wall and balanced shear and normal stresses at the free interface. The no slip condition at $y=0$ is

$$
u=\mathbf{v}=w=0 .
$$

The tangential and normal stress conditions at $y$ $=H(x, z, t)$, are given by 


$$
\begin{aligned}
& u_{y}=\sigma_{x}, \\
& w_{y}=\sigma_{z}, \\
& \Delta p=-\mathcal{C} \nabla^{2} H,
\end{aligned}
$$

where $\mathcal{C} \equiv \varepsilon^{2} \sigma_{m}^{*} / \Pi^{*}$, in which $\sigma_{m}^{*} / \Pi^{*}$ is typically of order one or less (unless the driving force $\Pi^{*}$ is so small that spreading is no longer dominated by Marangoni stresses). This dimensionless number is related to the capillary number $\mathrm{Ca}=\mu^{*} U^{*} / \sigma_{m}^{*}$ through the relation $\mathcal{C}=\varepsilon^{3} / \mathrm{Ca}$. The capillary number reflects the balance between Marangoni forces which favor more interface and capillary forces which minimize the amount of interface. In determining the overall scaling for the capillary pressure, $p$, the dimensionless value of the surface tension is approximated by $\sigma_{m} / \Pi$, wherein contributions to the absolute surface tension arising from $\Delta \sigma$ are ignored. Despite the fact that $\mathcal{C}$ is of order $\varepsilon^{2}$, derivatives of the free surface curvature can achieve magnitudes of $O\left(\varepsilon^{-2}\right)$ at the surfactant leading edge; therefore, capillary contributions cannot be uniformly neglected. ${ }^{26}$ The capillary terms also help smooth any cusplike features in the spreading profiles eliminating numerical difficulties.

The kinematic boundary condition at the liquid surface, $\mathrm{v}_{s}=d H / d t$, where $\mathrm{v}_{s}$ represents the vertical surface velocity at $y=H$, can be expressed in terms of the fluid flux as

$$
H_{t}+\left(H u_{\mathrm{avg}}\right)_{x}+\left(H w_{\mathrm{avg}}\right)_{z}=0,
$$

where $u_{\text {avg }}$ and $w_{\text {avg }}$ represent the streamwise and transverse height averaged velocities. The subscript $t$ denotes partial differentiation with respect to time. This equation determines the spatiotemporal profile of the spreading liquid layer in the presence of surfactant. Similarly, mass conservation of the insoluble surfactant is expressed by

$$
\Gamma_{t}+\left(\Gamma u_{s}\right)_{x}+\left(\Gamma w_{s}\right)_{z}=\frac{1}{\operatorname{Pe}_{s}}\left(\Gamma_{x x}+\Gamma_{z z}\right),
$$

where $\Gamma^{*}$ has been scaled by $\Gamma_{m}^{*}$ (the surface concentration corresponding to a surface tension of $\sigma_{m}^{*}$ ) and $u_{s}$ and $w_{s}$ represent the horizontal and transverse velocities of the liquid layer at $y=H(x, z, t)$. The modified surface Peclet number which appears in Eq. (9) is defined by $\mathrm{Pe}_{s}$ $\equiv\left(U^{*} L_{0}^{*}\right) / \mathcal{D}_{s}^{*}=\left(\Pi_{0}^{*} H_{0}^{*}\right) / \mu^{*} \mathcal{D}_{s}^{*}$, where $\mathcal{D}_{s}^{*}$ is the surface diffusion coefficient of the surfactant along the air-liquid interface. This dimensionless quantity represents the ratio of surfactant transport by Marangoni convection to that by surface diffusion. Although this ratio is typically very large, its inclusion locates more accurately the position of the surfactant front and improves the smoothness of the numerical profiles. In what follows, we focus on the large scale dynamics which reflect the balance of viscous and Marangoni forces. Capillary forces and surface diffusion merely act as corrections which smooth the shape of the spreading film in the thinned region near the source and the shocklike region near the leading edge.

Integration of Eqs. (2) and (4) subject to the boundary conditions in Eqs. (5)-(7) yields the dimensionless streamwise and transverse velocity field,

$$
[u, w](x, y, z, t)=y \nabla \sigma-\mathcal{C} \frac{y(y-2 H)}{2} \nabla^{3} H .
$$

The velocity profiles contain a simple shear term due to the Marangoni surface stress and a Poiseuille-type term due to capillary driven flow. Substitution of the height-averaged and surface velocities of the spreading film into the Eqs. (8) and (9) yields the two evolution equations for $H(x, z, t)$ and $\Gamma(x, z, t)$, namely,

$$
\begin{aligned}
& H_{t}+\frac{1}{2} \nabla \cdot\left(H^{2} \nabla \sigma\right)+\frac{\mathcal{C}}{3} \nabla \cdot\left(H^{3} \nabla^{3} H\right)=0, \\
& \Gamma_{t}+\nabla \cdot(\Gamma H \nabla \sigma)-\frac{1}{\mathrm{Pe}_{s}} \nabla^{2} \Gamma+\frac{\mathcal{C}}{2} \nabla \cdot\left(\Gamma H^{2} \nabla^{3} H\right)=0 .
\end{aligned}
$$

Since the surfactant molecules are free to spread across an unbounded surface, the likely surface configuration describing the expanding surfactant film is a gaseous monolayer. The dimensionless constitutive equation required to close this pair of equations is chosen to be $\sigma(\Gamma)=1-\Gamma$, the equation of state describing an ideal gas of surfactant molecules. $^{27}$

\section{B. Transient growth analysis}

\section{Base state}

We first consider the one-dimensional spreading dynamics in the absence of disturbances. The monolayer spreads spontaneously to produce a shear stress in the $x$ direction which deforms the thin viscous film. Equations (11) and (12) reduce to the form

$$
\begin{aligned}
& H_{o t}=\frac{1}{2}\left(H_{o}^{2} \Gamma_{o x}\right)_{x}-\frac{\mathcal{C}}{3}\left(H_{o}^{3} H_{o x x x}\right)_{x}, \\
& \Gamma_{o t}=\left(\Gamma_{o} H_{o} \Gamma_{o x}\right)_{x}+\frac{1}{\mathrm{Pe}_{s}} \Gamma_{o x x}-\frac{\mathcal{C}}{2}\left(\Gamma_{o} H_{o}^{2} H_{o x x x}\right)_{x} .
\end{aligned}
$$

The subscript "o" will henceforth designate the onedimensional solutions to these base state equations. Since Marangoni driven spreading is rather rapid, it is computationally more efficient to introduce a stretched horizontal coordinate, $\xi$, whose overall length is determined by the leading edge of the surfactant monolayer. For a finite amount of insoluble surfactant spreading in a one-dimensional geometry, a simple scaling analysis ${ }^{5,28}$ shows that the leading edge advances in time as $L(t)=t^{1 / 3}$. The following transformations therefore describe the spreading process in stretched coordinates:

$$
\begin{aligned}
& \xi=x / L(\tau), \quad H_{o}(x, t)=h_{o}(\xi, \tau), \\
& \Gamma_{o}(x, t)=\frac{g_{o}(\xi, \tau)}{L(\tau)}, \quad \text { where } \tau=t .
\end{aligned}
$$

The scaling for $\Gamma_{o}$ is determined from mass conservation of surfactant in a coordinate system whose horizontal extent is stretching with time. Insertion of the above transformations into Eqs. (13) and (14) yields

$$
\tau h_{o \tau}=\frac{1}{3} \xi h_{o \xi}+\frac{1}{2}\left(h_{o}^{2} g_{o \xi}\right)_{\xi}-\frac{\mathcal{C}}{3 \tau^{1 / 3}}\left(h_{o}^{3} h_{o \xi \xi \xi}\right)_{\xi},
$$




$$
\begin{aligned}
\tau g_{o \tau}= & \frac{1}{3}\left(\xi g_{o}\right)_{\xi}+\left(g_{o} h_{o} g_{o \xi}\right)_{\xi}+\frac{\tau^{1 / 3}}{\mathrm{Pe}_{s}} g_{o \xi \xi} \\
& -\frac{\mathcal{C}}{2 \tau^{1 / 3}}\left(g_{o} h_{o}^{2} h_{o \xi \xi \xi}\right)_{\xi} \cdot
\end{aligned}
$$

Equations (16) and (17) are solved subject to the following boundary conditions:

$$
\begin{array}{ll}
h_{o \xi}(0, \tau)=0, & h_{o \xi \xi \xi}(0, \tau)=0, \quad \text { and } g_{o \xi}(0, \tau)=0, \\
h_{o}(\infty, \tau)=1, & h_{o \xi}(\infty, \tau)=0, \quad \text { and } \quad g_{o}(\infty, \tau)=0 .
\end{array}
$$

Equation (18) represents symmetry and a no-flux condition about the origin while Eq. (19) describes the recovery of undisturbed conditions far downstream of the spreading monolayer.

\section{Disturbance equations}

The linearized version of Eqs. (11) and (12) can be used to determine the sensitivity of the system to small disturbances in the film thickness and surfactant concentration,

$$
\begin{aligned}
\widetilde{H}_{t}= & \frac{1}{2}\left(H_{o}^{2} \widetilde{\Gamma}_{x}+2 H_{o} \Gamma_{o x} \widetilde{H}\right)_{x}+\frac{1}{2} H_{o}^{2} \widetilde{\Gamma}_{z z}-\frac{\mathcal{C}}{3}\left[\left(H_{o}^{3} \widetilde{H}_{x x x}\right.\right. \\
& \left.\left.+3 H_{o}^{2} H_{o x x x} \widetilde{H}\right)_{x}+\left(H_{o}^{3}\right)_{x} \widetilde{H}_{x z z}+2 H_{o}^{3} \widetilde{H}_{x x z z}+H_{o}^{3} \widetilde{H}_{z z z z}\right], \\
\widetilde{\Gamma}_{t}= & \left(\Gamma_{o} \Gamma_{o x} \widetilde{H}+H_{o} \Gamma_{o x} \widetilde{\Gamma}+\Gamma_{o} H_{o} \widetilde{\Gamma}_{x}\right)_{x}+\frac{1}{\mathrm{Pe}_{s}}\left(\widetilde{\Gamma}_{x x}+\widetilde{\Gamma}_{z z}\right) \\
& +\Gamma_{o} H_{o} \widetilde{\Gamma}_{z z}-\frac{\mathcal{C}}{2}\left[\left(\Gamma_{o} H_{o}^{2} \widetilde{H}_{x x x}+2 \Gamma_{o} H_{o} H_{o x x x} \widetilde{H}\right.\right. \\
& \left.\left.+H_{o}^{2} H_{o x x x} \widetilde{\Gamma}\right)_{x}\right]-\frac{\mathcal{C}}{2}\left[\left(\Gamma_{o} H_{o}^{2}\right)_{x} \widetilde{H}_{x z z}+2 \Gamma_{o} H_{o}^{2} \widetilde{H}_{x x z z}\right. \\
& \left.+\Gamma_{o} H_{o}^{2} \widetilde{H}_{z z z z}\right] .
\end{aligned}
$$

The quantities decorated with "tilde" represent small deviations from the unperturbed solutions, $H_{o}$ and $\Gamma_{o}$. Since the base states are strictly one-dimensional and depend only on the $x$ coordinate, the coefficients preceding $\widetilde{H}$ and $\widetilde{\Gamma}$ (or their derivatives) in Eqs. (20) and (21) are independent of $z$. The disturbance functions can therefore be Fourier decomposed into the form

$$
(\widetilde{H}, \widetilde{\Gamma})(x, z, t)=(\Psi, \Phi)(x, t) e^{i K z} .
$$

This product describes a spatially inhomogeneous, time variant disturbance waveform in the streamwise direction of periodicity $2 \pi / K$ in the transverse direction. Substitution of this form into Eqs. (20) and (21) produces a coupled set of linear equations describing the evolution of two-dimensional disturbances,

$$
\begin{aligned}
\Psi_{t}= & \frac{1}{2}\left(H_{o}^{2} \Phi_{x}+2 H_{o} \Gamma_{o x} \Psi\right)_{x}-\frac{K^{2}}{2} H_{o}^{2} \Phi-\frac{\mathcal{C}}{3}\left[\left(H_{o}^{3} \Psi_{x x x}\right.\right. \\
& \left.+3 H_{o}^{2} H_{o x x x} \Psi\right)_{x}-K^{2}\left(\left(H_{o}^{3}\right)_{x} \Psi_{x}+2 H_{o}^{3} \Psi_{x x}\right) \\
& \left.+K^{4} H_{o}^{3} \Psi\right],
\end{aligned}
$$

$$
\begin{aligned}
\Phi_{t}= & \left(\Gamma_{o} \Gamma_{o x} \Psi+H_{o} \Gamma_{o x} \Phi+\Gamma_{o} H_{o} \Phi_{x}\right)_{x}-K^{2} \Gamma_{o} H_{o} \Phi \\
& +\frac{1}{\operatorname{Pe}_{s}}\left(\Phi_{x x}-K^{2} \Phi\right)-\frac{\mathcal{C}}{2}\left(\Gamma_{o} H_{o}^{2} \widetilde{H}_{x x x}\right. \\
& \left.+2 \Gamma_{o} H_{o} H_{o x x x} \widetilde{H}+H_{o}^{2} H_{o x x x} \widetilde{G}\right)_{x} \\
& -\frac{\mathcal{C}}{2}\left(-K^{2}\left(\left(\Gamma_{o} H_{o}^{2}\right)_{x} \Psi_{x}+2 \Gamma_{o} H_{o}^{2} \Psi_{x x}\right)+K^{4} \Gamma_{o} H_{o}^{2} \Psi\right) .
\end{aligned}
$$

As before, it proves computationally efficient to stretch the horizontal coordinate to lie coincident with the leading edge of the surfactant monolayer. This transformation also rescales the disturbances

$$
\Psi(x, t)=\psi(\xi, \tau) \quad \text { and } \Phi(x, t)=\frac{\phi(\xi, \tau)}{\tau^{1 / 3}} .
$$

Substitution of the transformations given by Eqs. (15) and (25) into Eqs. (23) and (24) yields the final stretched form of the equations to be solved,

$$
\begin{aligned}
\tau \psi_{\tau}=\mathcal{L}_{1}[\psi, \phi]= & \frac{1}{3} \xi \psi_{\xi}+\frac{1}{2}\left(h_{o}^{2} \phi_{\xi}+2 h_{o} g_{o \xi} \psi\right)_{\xi} \\
& -\frac{\left(K \tau^{1 / 3}\right)^{2}}{2} h_{o}^{2} \phi-\frac{\mathcal{C}}{3 \tau^{1 / 3}} \\
& \times\left[\left(h_{o}^{3} \psi_{\xi \xi \xi}+3 h_{o}^{2} h_{o \xi \xi \xi} \psi\right)_{\xi}\right] \\
& -\frac{\mathcal{C}}{3 \tau^{1 / 3}}\left[-\left(K \tau^{1 / 3}\right)^{2}\left(\left(h_{o}^{3}\right)_{\xi} \psi_{\xi}+2 h_{o}^{3} \psi_{\xi \xi}\right)\right. \\
& \left.+\left(K \tau^{1 / 3}\right)^{4} h_{o}^{3} \psi\right], \\
\tau \phi_{\tau}=\mathcal{L}_{2}[\psi, \phi]= & \frac{1}{3}(\xi \phi)_{\xi}+\left(g_{o} g_{o \xi} \psi+h_{o} g_{o \xi} \phi+h_{o} g_{o} \phi_{\xi}\right)_{\xi} \\
& -\left(K \tau^{1 / 3}\right)^{2} h_{o} g_{o} \phi+\frac{\tau^{1 / 3}}{\mathrm{Pe}_{s}}\left(\phi_{\xi \xi}-\left(K \tau^{1 / 3}\right)^{2} \phi\right) \\
& -\frac{\mathcal{C}}{2 \tau^{1 / 3}}\left[\left(g_{o} h_{o}^{2} \psi_{\xi \xi \xi}+2 g_{o} h_{o} h_{o \xi \xi \xi} \psi\right.\right. \\
& \left.\left.+h_{o}^{2} h_{o \xi \xi \xi} \phi\right)_{\xi}\right]-\frac{\mathcal{C}}{2 \tau^{1 / 3}} \\
& \times\left[-\left(K \tau^{1 / 3}\right)^{2}\left(\left(g_{o} h_{o}^{2}\right)_{\xi} \psi_{\xi}+2 g_{o} h_{o}^{2} \psi_{\xi \xi}\right)\right. \\
& \left.+\left(K \tau^{1 / 3}\right)^{4} g_{o} h_{o}^{2} \psi\right] .
\end{aligned}
$$




\section{Definitions describing transient amplification and growth}

The temporal evolution of the base state is strongly influenced by the initial shear stress and the amount of surfactant available for spreading. These variables depend, of course, on the choice of initial and boundary conditions. Since the finite mass of surfactant spreads to cover an ever larger area, the dominant driving force for spreading, which is the surface shear stress, commences with a large value. It eventually decays to zero when the surfactant has completely covered all the available surface area. At early times, the base states change rapidly in response to the large shear stress. As time increases, they decelerate toward a quasisteady form. The strong temporal response at early times precludes a straightforward analysis via separation of variables. How is the criterion for stability determined in such cases, since the growth or decay of disturbances is only meaningful in reference to the growth or decay of the evolving base state? As Shen ${ }^{17}$ first observed in his study of time dependent parallel shear flow, if a disturbance decreases in time but the base state decreases at a faster rate, then the disturbance will appear amplified in time. Conversely, if a disturbance increases in time but the base state increases faster still, then the disturbance will appear to decay in time. In order to probe the sensitivity to disturbances of time variant base states, Shen introduced the concept of "momentary stability" and defined appropriate measures to quantify the stability of such systems. We define below similar measures extended to our two variable system and use these normalized quantifiers of amplification and rate of growth to examine the stability characteristics of a spreading monolayer.

As mentioned in the Introduction, the disturbance equations contain an additional complication. The product, $\mathcal{L}_{i} \mathcal{L}_{i}^{\dagger}$, where $i=1,2$ and $\mathcal{L}_{1}$ and $\mathcal{L}_{2}$ are expressed by Eqs. (26) and (27), is not self-adjoint, i.e., $\mathcal{L}_{i} \mathcal{L}_{i}^{\dagger} \neq \mathcal{L}_{i}^{\dagger} \mathcal{L}_{i}$. This is easily seen from the fact that the base states, $h_{o}$ and $g_{o}$, depend on the spatial coordinate $\xi$; therefore, derivative operations applied to products of a base state and a disturbance function are noncommutative. This property implies that even if the base states were time invariant (which they are not) and even if the largest real part of the eigenvalues of $\mathcal{L}_{1}$ and $\mathcal{L}_{2}$ was negative, the system might still experience large transient amplification of disturbances. ${ }^{19,29}$ The transient analysis we have carried out is specifically geared toward determining whether the early time behavior displays any such "momentary instability" in the shape of fingering patterns. We also briefly discuss results of calculations wherein the base states were frozen at their self-similar form and only then disturbances applied. Even under these quasisteady conditions, the system experienced large transient growth indicating the importance of non-normality in this system.

We next introduce several measures to quantify the time dependent amplification of disturbances. The "mechanical energy" contained in either the base or disturbance states is given by

$$
E_{q} \equiv \frac{1}{2} \int_{0}^{\infty} q^{2}(\xi, \tau) d \xi, \text { where } q=\psi, \phi, h_{o}, g_{o}
$$

Of physical importance is the relative energy contained in an applied disturbance to that contained in the reference base state at time $\tau$, namely,

$$
M_{i}(\tau) \equiv \frac{E_{i}(\tau)}{E_{j}(\tau)}, \text { where }(i, j)=\left(\psi, h_{o}\right) \text { or }\left(\phi, g_{o}\right) .
$$

In our present study, we wish to determine the sensitivity of the spreading dynamics to small disturbances applied at time $\tau_{o} .{ }^{30}$ We therefore define an "amplification ratio" which describes the relative energy contained in the disturbed flow at time $\tau$ normalized by the relative energy contained in the initial disturbance at time $\tau_{o}$ according to

$$
G_{i}(\tau) \equiv \frac{M_{i}(\tau)}{M_{i}\left(\tau_{0}\right)}, \text { where }(i, j)=\left(\psi, h_{o}\right) \text { or }\left(\phi, g_{o}\right) .
$$

Equation (32) can be decomposed more conveniently into the ratio of normalized amplification factors for the individual base flow and disturbance contributions,

$$
\begin{aligned}
G_{i}=\left[\frac{E_{i}(\tau)}{E_{i}\left(\tau_{0}\right)}\right] /\left[\frac{E_{j}(\tau)}{E_{j}\left(\tau_{0}\right)}\right]= & \hat{G}_{i}(\tau) / \hat{G}_{j}(\tau), \\
& \text { where }(i, j)=\left(\psi, h_{o}\right) \text { or }\left(\phi, g_{o}\right) .
\end{aligned}
$$

With these definitions, the criterion for "momentary stability" of an unsteady base state, according to Shen, ${ }^{17,18}$ is determined by

$$
\begin{aligned}
\Omega_{i} \equiv \frac{1}{G_{i}} \frac{d G_{i}}{d \tau} & =\frac{1}{\hat{G}_{i}} \frac{d \hat{G}_{i}}{d \tau}-\frac{1}{\hat{G}_{j}} \frac{d \hat{G}_{j}}{d \tau} \\
& =\omega_{i}-\omega_{j}, \\
\text { where }(i, j) & =\left(\psi, h_{o}\right) \text { or }\left(\phi, g_{o}\right) .
\end{aligned}
$$

The quantities $\omega_{i}$ and $\omega_{j}$ are the normalized rates of energy production/removal associated with the perturbations and the unsteady base state, respectively. $\Omega_{i}<0$ defines momentary stability while $\Omega_{i}>0$ defines momentary instability. Equation (34) provides a measure of the degree of dominance of perturbations over the unsteady base state. The asymptotic stability of an unsteady base state is determined from

$$
\begin{aligned}
\Omega_{i} & \equiv \frac{1}{G_{i}} \frac{d G_{i}}{d \tau} \leqslant 0 \quad \text { as } \tau \rightarrow \infty, \\
& =\omega_{i}-\omega_{j} \leqslant 0 \text { where }(i, j)=\left(\psi, h_{o}\right) \text { or }\left(\phi, g_{o}\right) .
\end{aligned}
$$

For systems in which the rate of change of the base state as $t \rightarrow \infty$ becomes negligible in comparison to that of disturbances ${ }^{19,20}$ (i.e., $\omega_{j} \ll \omega_{i}$ ), the disturbance function assumes exponential form, $e^{\beta \tau}$, and the quantity $\omega_{\psi} / 2$ $=\left(\int_{0}^{\infty} \psi \psi_{\tau} d \xi\right) /\left(\int_{0}^{\infty} \psi^{2} d \xi\right)=\beta$, consistent with the definition of the quasistatic growth rate.

\section{Numerical procedure}

Our computations were performed using the method of lines ${ }^{31}$ which relies on second-order centered differences for the spatial derivatives and a fully implicit Gear's method for the time integration. ${ }^{32}$ The input values for the dimensionless 
quantities, $\mathcal{C}, \mathrm{Pe}_{s}$, and $K$, are specified at the start of the numerical integration. The number of grid points used in the computations varied between 201 and 801 depending on the film shape obtained for different choices of $\mathcal{C}$ and $\mathrm{Pe}_{s}$. Sharper fronts required more mesh points to resolve the entire shape of the spreading film from the source to the leading edge. Convergence of the solutions was checked via refinement of the spatial grid.

The sensitivity of the spreading process to infinitesimal disturbances in the film thickness or surfactant concentration is determined via the simultaneous solution of Eqs. (16), (17), (26), and (27). For most of the runs, integration was commenced at $\tau_{0}=1$ from a set of fixed initial conditions. The results depend to some degree on the choice of initial conditions. Two of the most important choices involve the shape of the initial surfactant distribution (which determines the initial shear stress) and the location at which the disturbances are applied. We have investigated several parameter sets in an effort to determine the spectrum of spreading behavior and the source of digitation. ${ }^{24,25,33}$ The choice discussed below reflects the common response of the system and demonstrates the most important features of the spreading process. We concentrate on the following set of initial conditions in analyzing the stability of the time dependent base states:

$h_{0}(\xi, 1)=1$,

$g_{o}(\xi, 1)=g_{o}^{\max }\left[1-\tanh \left(\mathrm{A}\left(\xi-\xi_{o}\right)\right)\right]$

and

$\psi(\xi, 1)=\phi(\xi, 1)=e^{-\mathrm{B}\left(\xi-\xi_{s}\right)^{2}}$.

The initial film thickness and surfactant concentration profiles described by Eq. (36) correspond to an initially flat liquid layer supporting a steplike concentration profile that smoothly vanishes to zero near $\xi_{o}$. This distribution function mimics the experimental situation in which a uniform patch of surfactant is deposited on a thin liquid layer. The tanh contribution smooths the edge of the distribution to prevent discontinuities in the profile. The initial disturbances described by Eq. (37) are Gaussian distributed functions centered at $\xi=\xi_{s}$, a position which lies ahead of the initial surfactant monolayer. Although not reported here, we have also studied disturbances located behind $\xi_{o}$. These produced much smaller effects on the spreading dynamics but a similar response. Since Eqs. (26) and (27) are both linear in $\psi$ and $\phi$, the overall amplitude of $\psi(\xi, 1)$ and $\phi(\xi, 1)$ can be set to unity with no loss in generality. In this work, we chose the parameter set for initial conditions to be $g_{o}^{\max }=0.5, A=10$, $\xi_{o}=0.5, B=12$, and $\xi_{s}=0.7$. Other choices of initial conditions have confirmed the qualitative behavior described below.

In addition to the studies in which we have evolved the base states and disturbances simultaneously from $\tau_{o}=1$ in order to investigate the possibility of large energy production at early times, we also provide an illustrative example in which the base state was evolved through $\tau=5$ before a disturbance was applied. By this time, the base state has evolved into a self-similar form. ${ }^{25}$ The base flow profiles were first generated by numerical integration of Eqs. (16) and (17) subject to the initial conditions in Eqs. (36) with $g_{\mathrm{o}}^{\max }=0.5, A=10$, and $\xi_{o}=0.5$. The solutions were advanced to $\tau=5$ and then fixed, whereupon disturbances described by Eqs. (37) with $B=200$ and $\xi_{s}=1.6$ were applied starting from $\tau_{o}=5$. These disturbances coincided with the location of the maximum height in the shocklike structure which develops in the unperturbed film. Although a comprehensive study using this quasisteady approach requires a more complete study, the results shown here seem to confirm the behavior observed in the fully transient calculations.

\section{RESULTS AND DISCUSSION}

\section{A. Numerical results}

\section{Base state profiles}

Shown in Fig. 3 are the results of the base state solutions at an intermediate time, $\tau=5$, for the range $10^{-6} \leqslant \mathcal{C} \leqslant 10^{-4}$ and $5 \cdot 10^{2} \leqslant \mathrm{Pe}_{s} \leqslant 5 \cdot 10^{4}$. We have determined from the information provided in the literature ${ }^{7-12,34}$ that this range for $\mathcal{C}$ and $\mathrm{Pe}_{s}$ should bracket the experimental data. As seen in Figs. 3(a) and 3(c), the initially flat liquid layer with $h_{o}=1$ is deformed into a traveling waveform with a shocklike front and substantial thinning in the upstream region. Comparison of these profiles with Figs. 3(b) and 3(d) indicates that the surfactant concentration vanishes at the location where the steepened rim meets the undisturbed liquid layer. As expected from the physical mechanism responsible for the rapid spreading, an increase in $\mathrm{Pe}_{s}$ or a decrease in $\mathcal{C}$ produces a sharper front; however, changes in $\mathcal{C}$ or $\mathrm{Pe}_{s}$ by several orders of magnitude still produce base state profiles which are remarkably similar. Also evident from Figs. 3(b) and $3(\mathrm{~d})$ is the fact that the surfactant concentration, $g_{o}(\xi, \tau)$, is far less sensitive to changes in the values of $\mathcal{C}$ and $\mathrm{Pe}_{s}$. These results agree with previous numerical solutions for a spreading monolayer by Jensen and Grotberg. ${ }^{5}$

The profiles shown here represent the film shapes and concentration profiles after four shear times (since $\tau_{o}=1$ ). These shapes are characteristic of Marangoni driven flow in thin liquid films. For the parameter values used, these shapes maintain the same form for $\tau>5$. These profiles will be discussed again in a later section when we describe the stability of quasisteady base states. For the calculations described next, however, which treat the stability of the timedependent state, the base states and disturbances are evolved simultaneously from the instant the monolayer is deposited $\left(\tau_{o}=1\right)$. In this way, we monitor the evolution of disturbances applied at the moment the spreading commences. The early time base states (not shown), which resemble a compressed form of those shown in Fig. 3, are properly used to normalize the amplification ratio and rate of disturbance growth defined earlier in Sec. II B 3.

\section{Stability of time-dependent base state}

Figure 4(a) reports the amplification ratio, $G_{\Psi}(\tau)$, for various wave number disturbances with $\mathcal{C}=10^{-5}$ and $\mathrm{Pe}_{s}$ $=5000$. This ratio increases by over two orders in magnitude within one shear time. Such large amplification of small dis- 

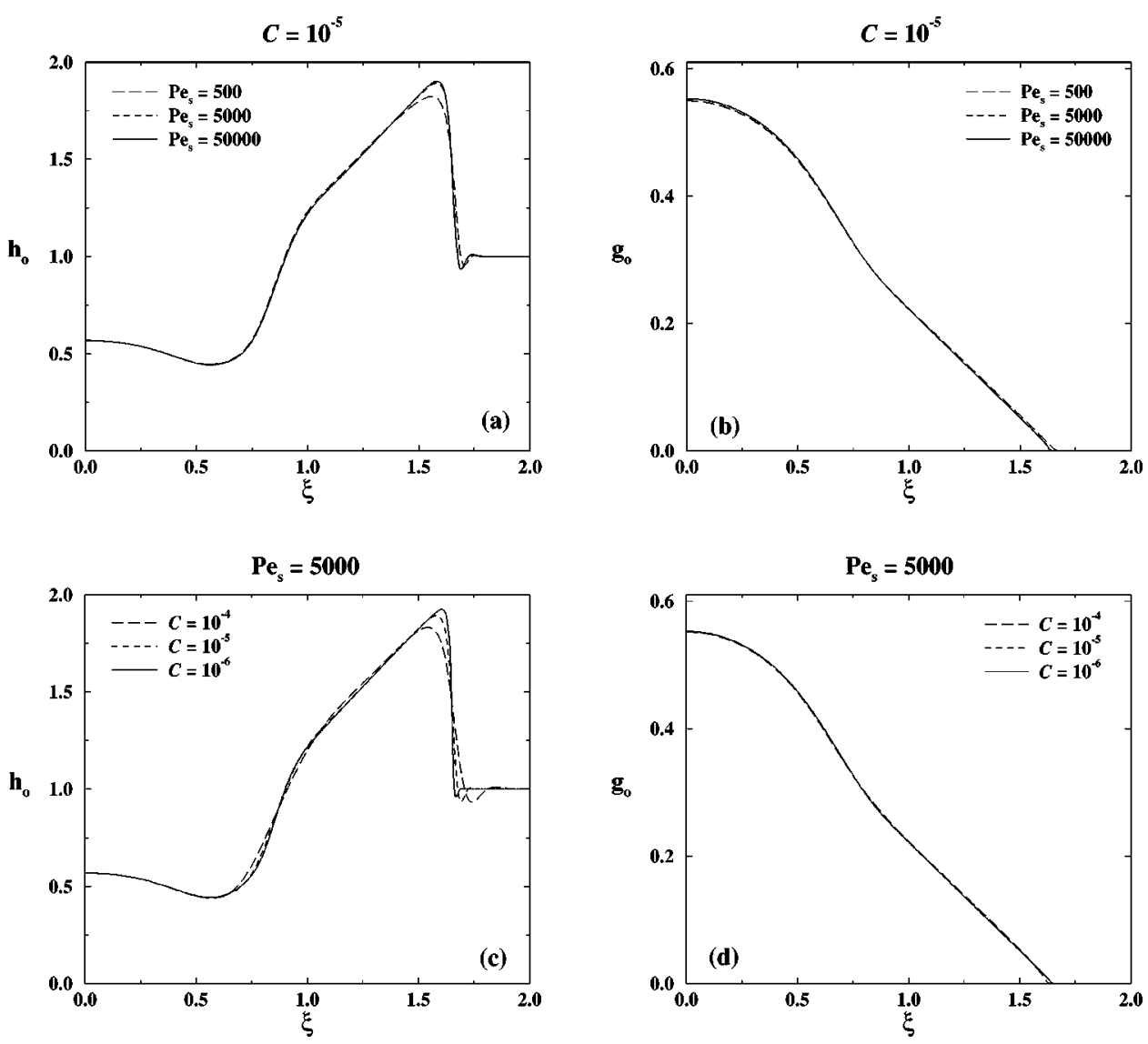

FIG. 3. Profiles showing the dependence of the unperturbed film thickness, $h_{o}(\xi, \tau)$, and surfactant concentration, $g_{o}(\xi, \tau)$, on $\mathrm{Pe}_{s}$ and $\mathcal{C}$ at $\tau$ $=5$. For (a) and (b), $\mathcal{C}=10^{-5}$. For (c) and (d), $\mathrm{Pe}_{s}=5000$ turbances has also been observed in viscous shear flows between parallel plates. ${ }^{35}$ Figure 4 (a) indicates that disturbances with large wave number dominate the response at early times but quickly decay. Disturbances of smaller wave number grow more slowly at first and with smaller amplitude but persist for longer periods of time. The disturbance corresponding to $K=0$, which has no periodicity in the transverse direction, survives for very long times although it too eventually decays to zero. We suspect this mode represents the same neutrally stable mode which was identified by the quasisteady linear stability analysis as the mode with the largest growth rate. $^{22-25}$ As shown in Fig. 4(b), the amplification ratio experienced by disturbances in the surfactant concentration, $G_{\Phi}$, is insignificant. Concentration variations do not directly influence the stability of the flow on a transient time scale. These small variations, however, strongly influence the film shape, which undergoes significant deformation at early times.

In Fig. 5 is shown the evolved shape of the disturbance functions, $\psi$ and $\phi$, plotted below the base state solution, $h_{o}$, for times $\tau=3$ and 5 and for $K=5, \mathcal{C}=10^{-5}$ and $\mathrm{Pe}_{s}$ $=5000$. The disturbances, which originate just ahead of the initial surfactant monolayer, are convected up and over the shocklike front, eventually lagging behind the moving front. (Although the disturbances seem to move backward in time, this is simply an artifact of the transformed coordinates since in the stretched variable the disturbances lag behind the faster moving front.) It is evident that the amplitude of the disturbance film thickness far exceeds that of the surfactant concentration. Figure 6 indicates more clearly the shape of the spreading film as it responds to an applied disturbance of $K=5$ during a time interval $1.40 \leqslant \tau \leqslant 11.0$. This three dimensional representation highlights the spatial and temporal evolution of the periodic surface corrugation. The disturbance is most strongly amplified in the vicinity of the shocklike front but as time evolves it falls behind the leading edge slowing considerably in the thinned portion of the film where its mobility is decreased. This $3 \mathrm{D}$ representation strongly resembles the fluid channeling or finger formation which accompanies the spreading of surfactant on thin liquid films as observed in many experiments.

More studies of this system of equations using other parameter values ${ }^{24,25,33}$ confirms that the transient growth observed in Fig. 4 can achieve even larger amplification ratios. For example, as shown in Fig. 7(a), placing the disturbance further ahead of the initial surfactant monolayer [by increasing $\xi_{s}$ in Eq. (37)] produces a larger amplification ratio. In this case, the spreading film has advanced to develop an even steeper rim which is apparently more vulnerable to lateral disturbances. Figures 7(b), 7(c) and 7(d) represent other trends we have observed. An increase in the initial shear stress, obtained by increasing $A$, or a sharpening of the shock-like rim, obtained by increasing $\mathrm{Pe}_{s}$ or decreasing $\mathcal{C}$, all produce a larger transient response reflected in $G_{\Psi}$. In addition, we have found that the $K=0$ mode maintains the largest amplification ratio at long times, eventually decaying to zero as $\tau \rightarrow \infty$. These results support our earlier predictions of asymptotic stability using a strictly modal analysis. ${ }^{22,23}$ For the reasons explained above, however, this analysis 

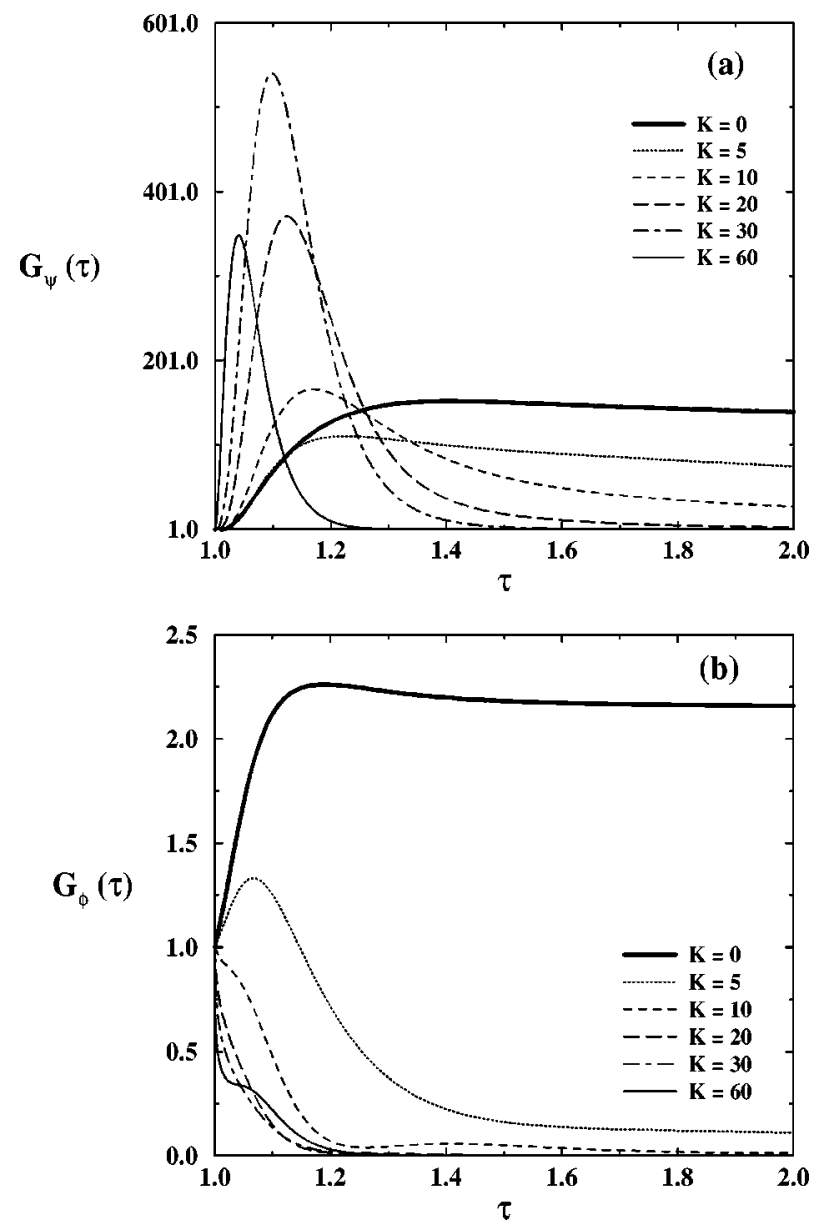

FIG. 4. Time evolution of the amplification ratio for disturbances in the film thickness and surfactant concentration as a function of wave number $K$. Relevant parameter values are $\mathrm{Pe}_{s}=5000$ and $\mathcal{C}=10^{-5}$. (a) $G_{\psi}(\tau)$ and (b) $G_{\phi}(\tau)$.

could not uncover the interesting transient behavior observed here.

It is also worthwhile to examine the growth rate of disturbances at onset. Figure 8 shows the normalized growth rate in the film thickness for various wave number disturbances. As seen more clearly in the magnified version in Fig. $8(\mathrm{a})$, shortly following the application of a disturbance, the flow counteracts its effect with a large stabilizing response. The system is unable to maintain this response and is rapidly "destabilized" until the transient growth dies away. We describe this response from a physical point of view in Sec. III B. The system returns to equilibrium at longer times as shown by Fig. 8(b). The growth rate in the disturbance surfactant concentration (not shown), $\Omega_{\Phi}$, exhibits almost identical behavior although the magnitude of the growth rate is far less.

\section{Stability of quasisteady base state}

As an additional consideration, we compare the above results in which the disturbances were applied at $\tau_{0}=1$ (the origin of time), with results obtained by freezing the base state once it has achieved self-similar form at $\tau_{0}=5$ and only then applying a disturbance. These base state solutions are
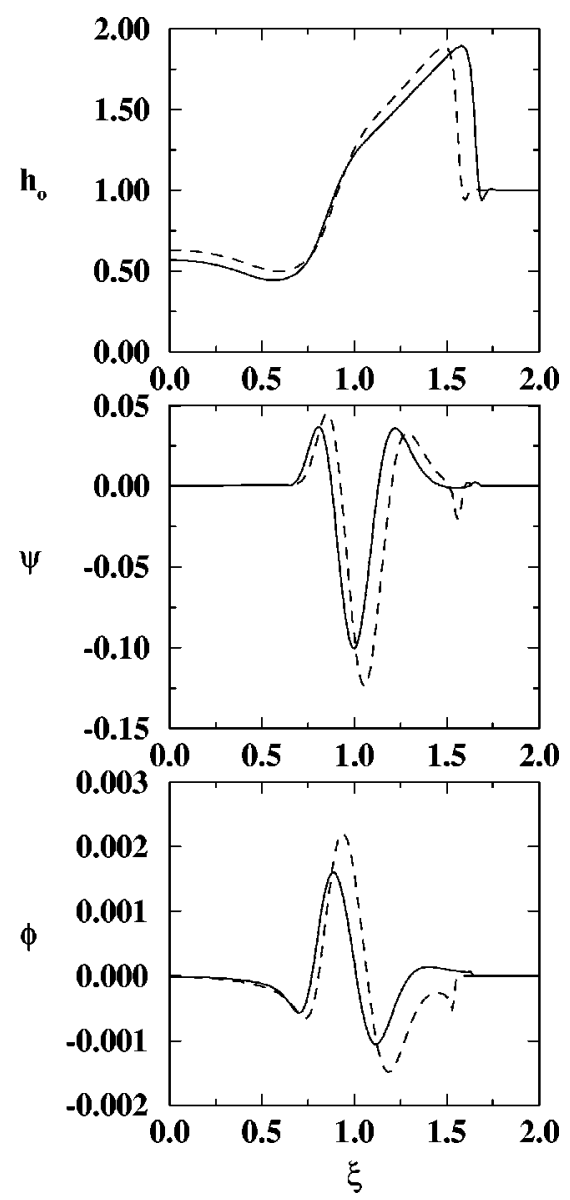

FIG. 5. Base state solution, $h_{o}(\xi, \tau)$, with evolved disturbances, $\psi$ and $\phi$, for $K=5, \mathrm{Pe}_{s}=5000$ and $\mathcal{C}=10^{-5}$ at two different times. The dashed lines represent $\tau=3$ while the solid lines represent $\tau=5$.

shown in Fig. 3 for $\mathrm{Pe}_{s}=5000$ and $\mathcal{C}=10^{-5}$. Figure 9 depicts the amplification ratios, $G_{\psi}$ and $G_{\phi}$, for various wave number disturbances. Despite the absence of any time dependence in the base states, there still exists significant transient amplification in the film thickness. Since the base state is no longer time variant in these calculations, the growth can only be attributed to the non-normality of the linearized operators governing the evolution of disturbances. As with the previous calculations, all the modes eventually decay. The response illustrated in Fig. 9 is qualitatively similar to that shown in Fig. 4; the large wave number disturbances grow most rapidly at early times but the smallest wave number ones persist for longer times.

\section{Relation to other fingering instabilities in spreading films}

A transient growth calculation ${ }^{36}$ has also been performed for another free surface flow involving the spreading of a thin viscous film down an inclined plane. In this example, there is only one variable to track, namely, the film thickness. In addition, there exists a traveling wave solution moving at constant speed down the plane which eliminates the problem of a time variant base state. The front of the falling film has been observed in many experiments to undergo a rivulet instability as first studied by Huppert. ${ }^{37}$ A straightfor- 


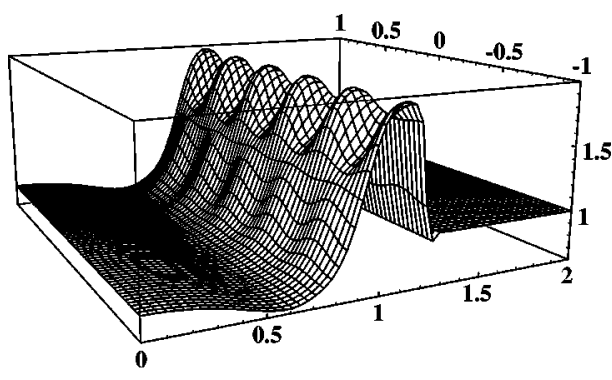

(a)

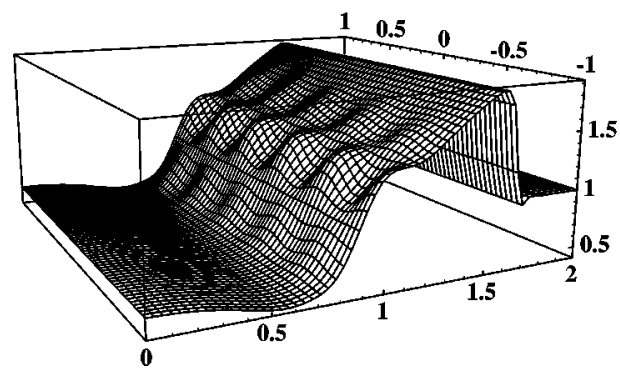

(c)

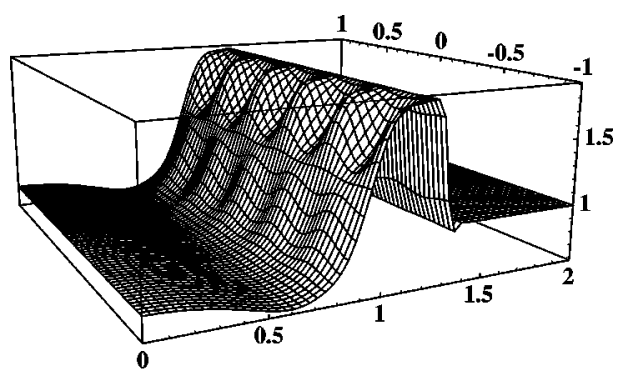

(b)

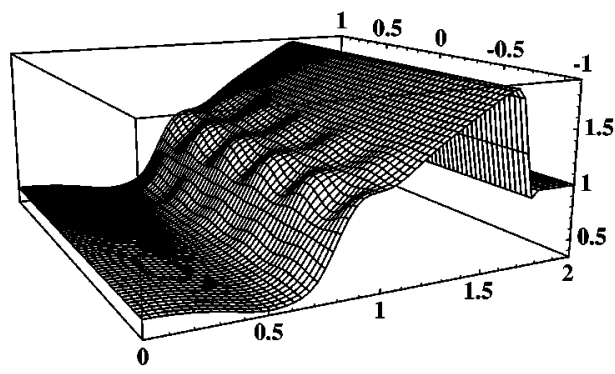

(d)
FIG. 6. 3-D representation of the shape of a spreading surfactant film in the presence of an applied disturbance with $K=5$. Relevant parameter values are $\mathrm{Pe}_{s}=5000$ and $\mathcal{C}=10^{-5}$. (a) $\tau$ $=1.4$, (b) $\tau=1.6$, (c) $\tau=5.0$ and (d) $\tau=11.0$. ward modal analysis has predicted the most dangerous mode observed experimentally. ${ }^{38,39}$ Since the base state is spatially inhomogeneous, however, there once again occurs the problem with nonnormality. The transient behavior was recently investigated in an effort to understand its evolution toward asymptotic instability. ${ }^{36}$ These calculations for the falling film show a short period of transient growth which quickly asymptotes to the exponential modes predicted by the linear stability analysis. When the falling film develops a significant capillary rim at the leading edge, the system reaches asymptotic instability rather quickly, which is probably why the modal analysis has successfully explained many experimental observations. This rapid approach to asymptotic behavior has also been found in an analogous problem with thermally driven films. ${ }^{40}$

These two free surface flows, however, are quite different. As a point of reference, the peak in the capillary rim which forms at the leading edge of the falling film is orders of magnitude larger than the thickness of the pre-existing liquid layer on the substrate. This produces a huge mobility
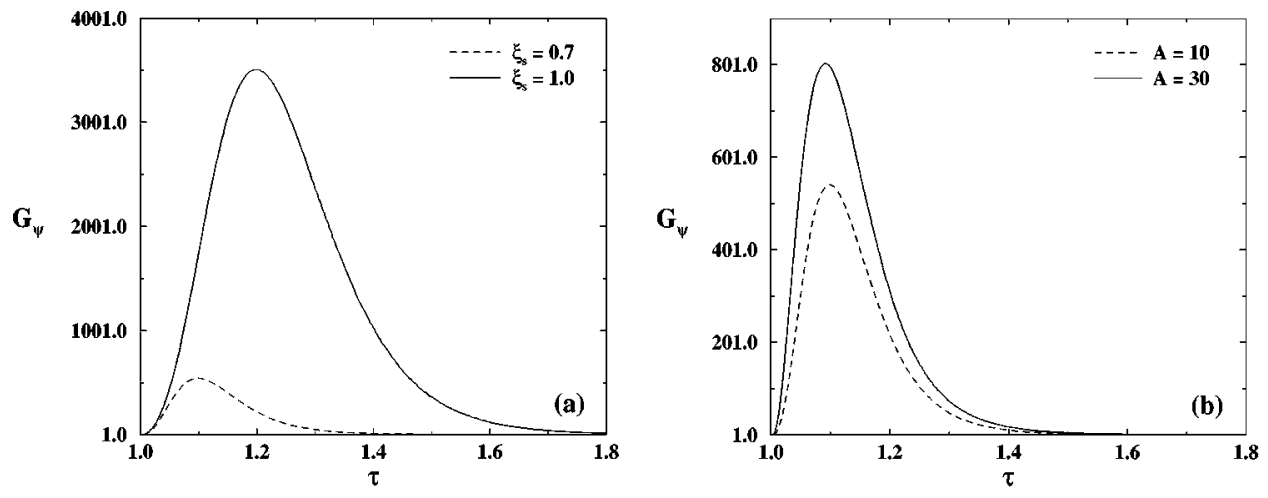

FIG. 7. Trends observed during the transient growth of disturbances in the film thickness with $K=30$ as a function of various input parameters. (a) $\mathrm{Pe}_{s}=5000, \mathcal{C}=10^{-5}$, and $A=10$, (b) $\mathrm{Pe}_{s}=5000, \mathcal{C}=10^{-5}$, and $\xi_{s}=0.7$, (c) $\mathcal{C}=10^{-5}, A=10$, and $\xi_{s}=0.7$, and (d) $\mathrm{Pe}_{s}=5000, A=10$, and $\xi_{s}=0.7$.

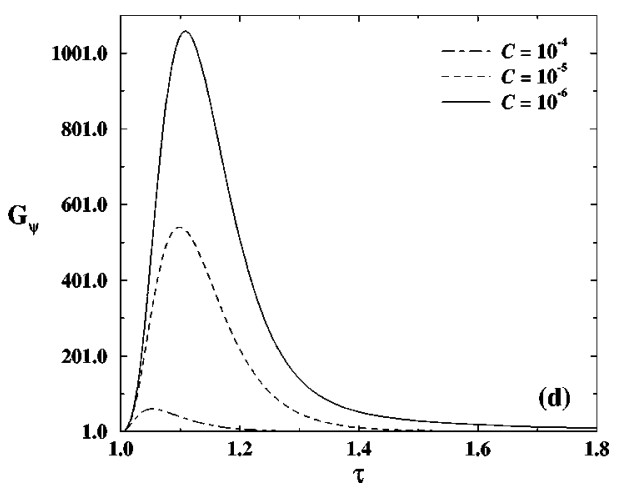



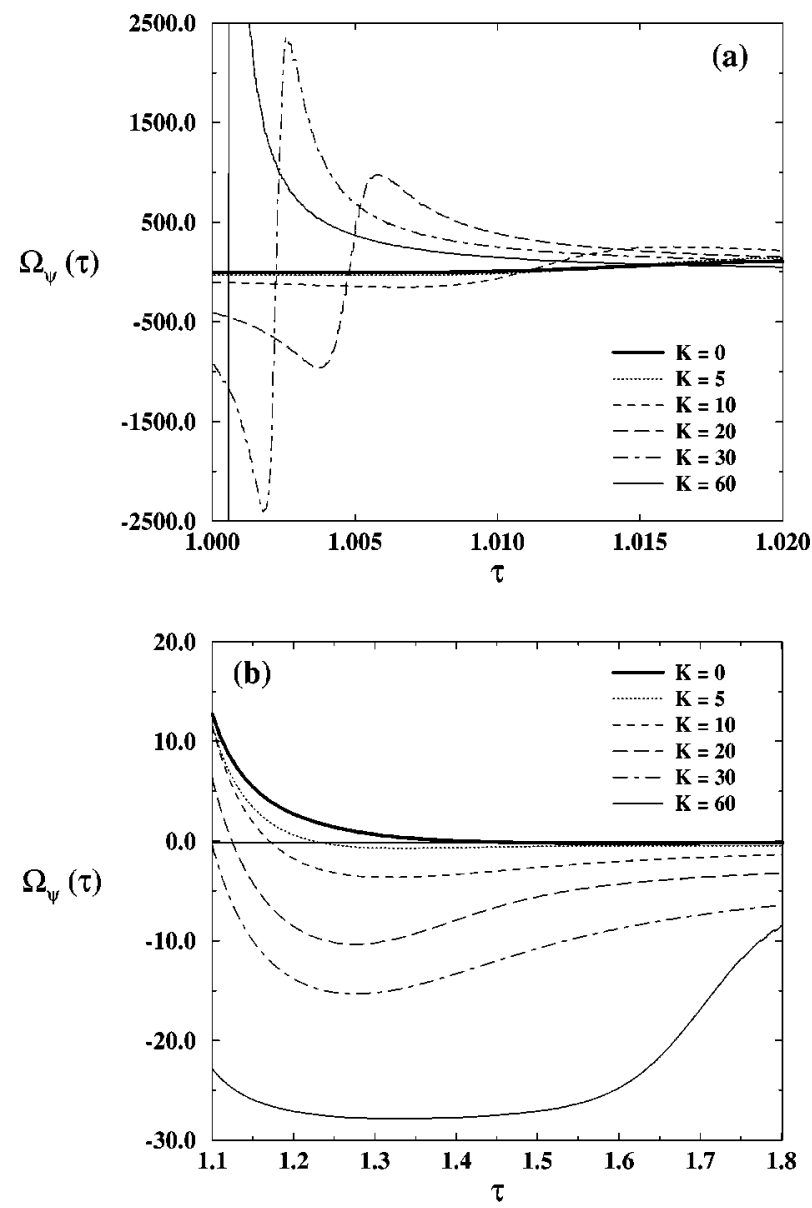

FIG. 8. Time evolution of the normalized rate of growth for disturbances in the film thickness, $\Omega_{\psi}$. Relevant parameter values are $\mathrm{Pe}_{s}=5000$ and $\mathcal{C}$ $=10^{-5}$. (a) Early time response. (b) Intermediate time response.

contrast at the leading edge and subsequent large transient growth. When the difference in film heights approaches the much smaller values obtained with the surfactant problem (in which the shocklike rim is at most a factor of 2 thicker than the initial liquid layer, see Fig. 3), the falling film exhibits no transient growth and asymptotic stability (see Fig. 11 in Ref. (36). By contrast, the surfactant film produces dendritic patterns even when the amplitude difference is less than 2 . In addition, the source of finger formation in these two flows is not the same, as indicated by the different shapes produced. The surfactant system always exhibits dendritic spreading patterns produced from a tip-splitting process. The falling film instability creates rivulet type fingers with no tipsplitting. Although the fingering mechanism for the surfactant problem is not completely understood, the following description serves to distinguish these flows. The falling film instability results from a combination of increased mobility and capillary breakup of the advancing rim. The rivulets form at the leading edge and race ahead of the majority of the spreading film. By contrast, the surfactant fingers first appear behind the advancing monolayer front where the film has thinned and where there exists a region with a constant concentration gradient. Although it has yet to be proven, the presence of dendritic patterns in the surfactant spreading problem strongly suggest a fingering mechanism driven by
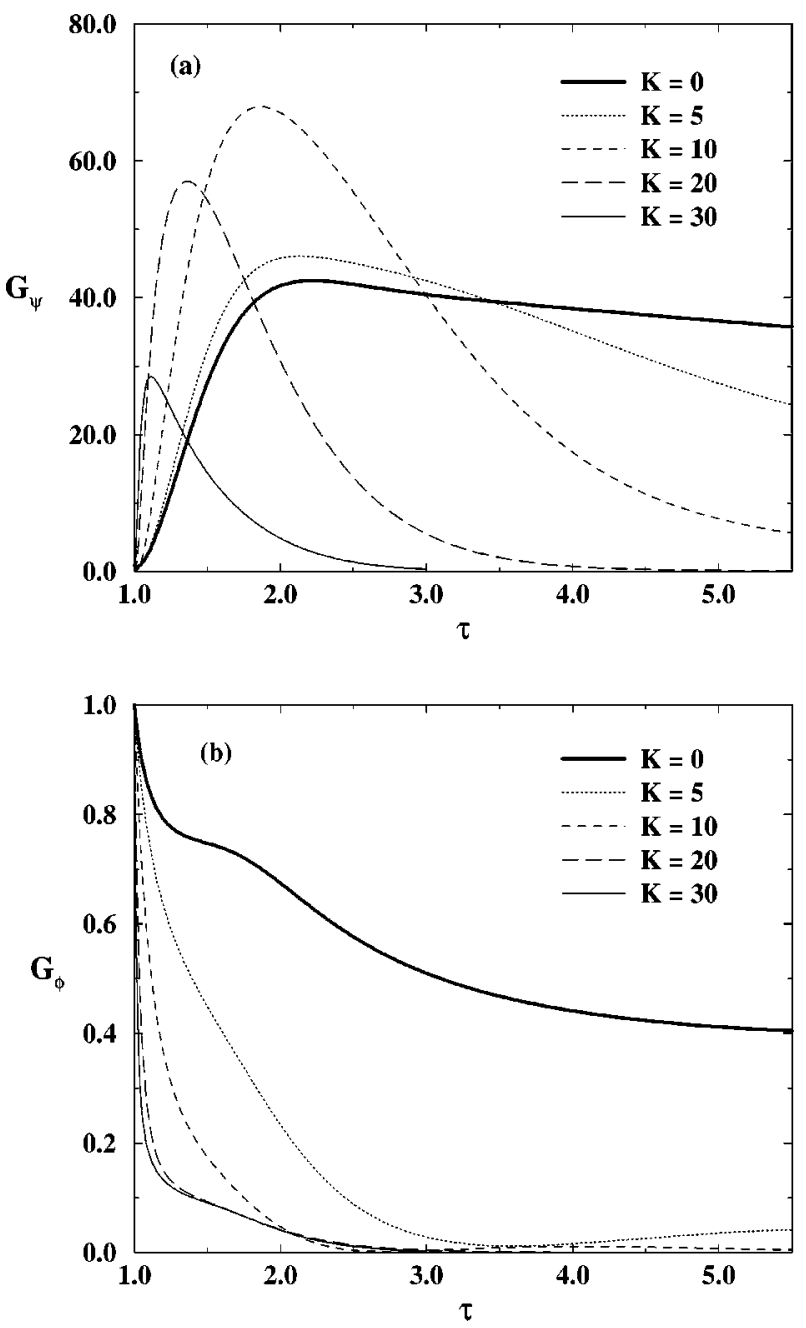

FIG. 9. Time evolution of the amplification ratio for disturbances in (a) the film thickness, $G_{\psi}(\tau)$, and (b) the surfactant concentration, $G_{\phi}(\tau)$, for various wave numbers, $K$. Base state is frozen at $\tau_{0}=5$. Relevant parameter values are $\mathrm{Pe}_{s}=5000$ and $\mathcal{C}=10^{-5}$.

Laplacian growth as described in the Introduction. Although both these systems possess non-normal linearized operators, the physical mechanisms for spreading and finger formation are very different in character.

\section{B. Proposed mechanism}

The momentary stability of a system is governed by the difference in the rate of energy growth between the disturbances and the unsteady base state as defined by Eq. (34). Since the amplification ratio of the disturbances is quite large and since the amplitudes of the decelerating base states decay in time, the criterion for "stability" is mostly determined by the behavior of $\omega_{\psi}$ and $\omega_{\phi}$. This can be seen by comparing the quantities $\omega_{\psi} / 2$ and $\omega_{h_{o}} / 2$ shown in Fig. 10(a) in which $\omega_{\psi} / 2$ exceeds $\omega_{h_{o}} / 2$ by several orders of magnitude. The normalized rate of growth of disturbances greatly exceeds that of the base state even at the earliest times. It is therefore meaningful to decompose the flow into a base state and a disturbance in order to analyze the momentary stability of the spreading process. 

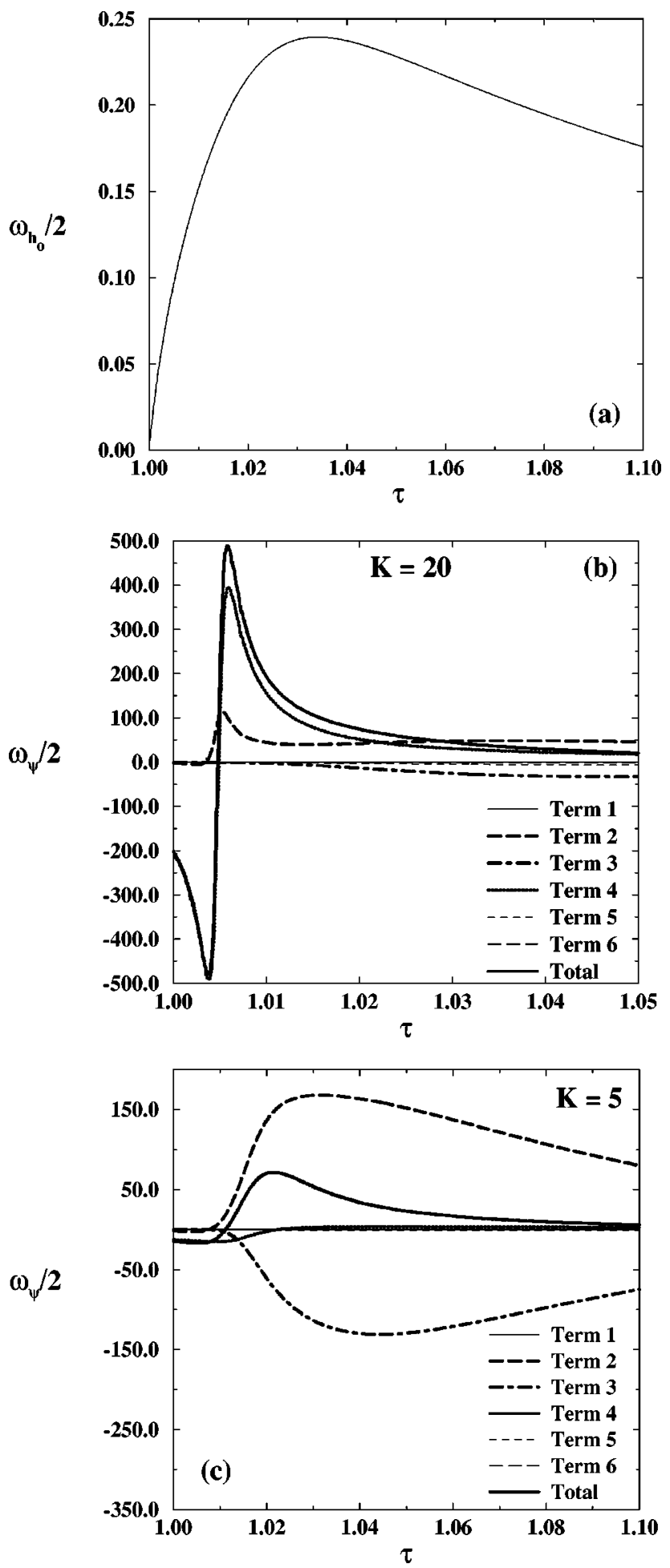

FIG. 10. Temporal evolution of the normalized rate of growth of the unsteady base state and its corresponding disturbance function for two different wave numbers. A physical description of each term can be found in Table I. (a) $\omega_{h_{o}} / 2$, (b) $\omega_{\psi} / 2$ for $K=20$, and (c) $\omega_{\psi} / 2$ for $K=5$.

To identify the largest destabilizing influences, we separate the rate of energy growth, $\omega_{\psi} / 2$, into six terms to monitor which forces produce or remove energy from the system as time increases. In Table I is listed each component of $\omega_{\psi} / 2$ along with a brief descriptor of its physical origin. These six quantities, including their summation, are plotted in Fig. 10 for two different values of the wave number ( $K$ $=20$ and 5). These two cases illustrate the difference in energy breakdown and the various competing physical mechanisms at large and small wave number. In both cases, the most dominant contributions (whether stabilizing or destabilizing) stem from terms 2,3 , and 4 . Terms 2 and 4 represent Marangoni convection of the fluid layer in the streamwise and transverse directions, respectively, while term 3 represents capillary flow driven by the film curvature of the base state and disturbance functions in the streamwise direction. Terms 5 and 6 , which describe additional capillary driven flow arising from the surface curvature in the transverse direction and the coupling of curvature in the streamwise and transverse directions, contribute very little to the overall dynamics. By comparing the curves in (b) and (c) of Fig. 10, it is evident that disturbances of smaller wave number produce larger destabilizing contributions to Marangoni convection in the streamwise direction, which are counteracted by larger stabilizing contributions to capillary flow in the streamwise direction. This arises because the surface shear stress establishes a thickened advancing front whose curvature is smoothed by the capillary terms. For large wave number disturbances, however, it is Marangoni convection in the transverse direction which is highly effective in destabilizing the flow, with little counterbalance provided by any other term. The main destabilizer at large wave numbers is therefore transverse Marangoni convection; the main destabilizer at small wave numbers is streamwise Marangoni flow.

From this analysis, what can we infer about the dynamics leading to finger formation as the wave number is varied? Let us first consider disturbances of small wavelength (large $K$ ) and assume that the disturbance functions $\psi$ and $\phi$ are in phase, as described by Eq. (22). The crests of the disturbance in the film thickness, initially placed ahead of the surfactant monolayer, are therefore laden with surfactant, while the troughs suffer an initial depletion. As the spreading begins, transverse Marangoni convection (term 4), and to a smaller degree streamwise Marangoni convection (term 2), quickly try to reestablish equilibrium and diminish the sinusoidal corrugation in film thickness and surfactant concentration. The crests undergo strong and rapid thinning in both the transverse and streamwise directions with subsequent thickening of the film in neighboring regions of higher surface tension. The redistribution of liquid and surfactant associated with this response is facilitated by contact with the oncoming shocklike rim which increases the mobility of the disturbance overall. The transverse redistribution of liquid from crests to troughs is thereby greatly enhanced and actually overshoots its ability to fill in the troughs. This overshoot produces thick liquid arteries in the regions formerly occupied by troughs. In the frame of the shocklike rim, the initial sinusoidal disturbance is observed to propagate over and behind the shocklike rim with a subsequent sideways redistribution of fluid. This flow behavior produces long striations in the streamwise direction throughout the entire film. The disturbance travels more slowly than the advancing front and eventually nestles closer to the thinned region of the spreading film, where its mobility is further decreased. This may explain why the fingering patterns observed experimentally 
TABLE I. Individual contributions comprising the rate of energy growth, $\omega_{\psi}$.

\begin{tabular}{lcc}
\hline \hline Terms & Expression & Physical mechanism \\
\hline 1 & $\int_{0}^{\infty} \frac{1}{6 \tau E_{\psi}}\left[\xi\left(\psi^{2}\right)_{\xi}\right] d \xi$ & $\begin{array}{c}\text { Streamwise flow due to coordinate } \\
\text { transformation } \xi=x / L(\tau)\end{array}$ \\
2 & $\int_{0}^{\infty} \frac{1}{2 \tau E_{\psi}}\left[\psi\left(h_{o}^{2} \phi_{\xi}+2 h_{o} g_{o \xi} \psi\right)_{\xi}\right] d \xi$ & Streamwise Marangoni flow \\
3 & $\int_{0}^{\infty}-\frac{\mathcal{C}}{3 \tau^{4 / 3} E_{\psi}}\left[\psi\left(h_{o}^{3} \psi_{\xi \xi \xi}+3 h_{o}^{2} h_{o \xi \xi \xi} \psi\right)_{\xi}\right] d \xi$ & Streamwise capillary flow \\
4 & $\int_{0}^{\infty}-\frac{\left(K \tau^{1 / 3}\right)^{2}}{2 \tau E_{\psi}}\left[h_{o}^{2} \psi \phi\right] d \xi$ & Transverse Marangoni flow \\
5 & $\int_{0}^{\infty} \frac{\mathcal{C}}{3 \tau^{4 / 3} E_{\psi}}\left(K \tau^{1 / 3}\right)^{2}\left[\psi\left(\left(h_{o}^{3}\right)_{\xi} \psi_{\xi}+2 h_{o}^{3} \psi_{\xi \xi}\right)\right] d \xi$ & Capillary flow from coupling of curvature \\
in streamwise and transverse directions
\end{tabular}

are mostly visible in the thinned portions of a spreading surfactant film. The capillary pressure arising from surface curvature in the streamwise and transverse directions (terms 5 and 6) always stabilizes the flow but its contribution is small by comparison.

For long wavelength disturbances (small $K$ ), the same forces described above come into play to redistribute fluid, except that the transverse concentration gradients are much smaller. Longer wavelength disturbances produce raised portions of the liquid film which contain more fluid. Marangoni stresses are therefore more effective in redistributing this fluid in the streamwise direction since the velocity for spreading increases with the local film thickness. For this reason, regions formerly occupied by the troughs begin to thicken in response to a transverse influx of fluid. The strong streamwise pull causes the spreading film to assume a steeper shocklike structure which is then counteracted by capillary forces which flatten the advancing rim. Nonetheless, disturbances with large wave number survive intact for a longer period and dominate the flow at late times. In both cases, as time increases the disturbance decays away since the finite amount of surfactant added to the spreading film must distribute itself over an increasingly larger spreading area thereby diminishing its influence, as is true for the base state also.

\section{CONCLUSION}

This transient growth study reveals the sensitivity of spreading surfactant coated film to small disturbances in the film height or surfactant distribution. The analysis supports the evolution of periodic structures in the direction transverse to the spreading process. The equations describing the spreading dynamics include Marangoni forces, capillarity, and surface diffusion. Our findings indicate that the rapidly spreading film is extremely sensitive to disturbances in the film thickness. Measures of the disturbance amplification ratio and growth rate at onset, indicate significant growth within a characteristic shear time. This large transient growth could very well excite a nonlinear response leading to finger formation.

Detailed study of the temporal evolution of disturbances reveals that large wave number disturbances are more desta- bilizing at early times while smaller wave number modes persist with large transient growth to later times, in agreement with our previous modal analysis. ${ }^{22-25}$ An energy decomposition reveals that there is a switch in the destabilizing agent between large and small $K$ disturbances. While the transverse Marangoni flux produces thickened arteries that grow into liquid channels for large $K$, the streamwise Marangoni flux produces similar channeling of the fluid for small $K$. In both cases, the destabilizing flow is resisted by streamwise capillary flow which is stabilizing at all times. Our 3D reconstructions provide strong evidence of fluid channeling and finger formation at early times following the deposition of a surfactant monolayer on a thin viscous film. In addition, recent results ${ }^{24}$ indicate that the inclusion of van der Waal's forces which promote film thinning enhance these disturbances to produce even more prominent film corrugation near the source. These and other similarities we have discussed between the experimental observations and numerical computations are encouraging.

\section{ACKNOWLEDGMENTS}

This work was supported by the NSF through a Research Initiation Award (CTS-9409579) and a Career Award (CTS9624776).

${ }^{1}$ B. Robertson, Pulmonary Surfactant (Elsevier, New York, 1984).

${ }^{2}$ D. L. Shapiro, Surfactant Replacement Therapy (Liss, New York, 1989).

${ }^{3}$ M. E. Borgas and J. B. Grotberg, "Monolayer flow on a thin film,' J. Fluid Mech. 193, 151 (1988).

${ }^{4}$ D. P. Gaver and J. B. Grotberg, “The dynamics of a localized surfactant on a thin film,', J. Fluid Mech. 213, 127 (1990).

${ }^{5}$ O. E. Jensen and J. B. Grotberg, "Insoluble surfactant spreading on a thin viscous film: Shock evolution and rupture,', J. Fluid Mech. 240, 259 (1992).

${ }^{6}$ A. Marmur and M. D. Lelah, "The spreading of aqueous surfactant solutions on glass,' Chem. Eng. Commun. 13, 133 (1981).

${ }^{7}$ S. M. Troian, X. L. Wu, and S. A. Safran, "Fingering instability in thin wetting films,' Phys. Rev. Lett. 62, 1496 (1989).

${ }^{8}$ A. M. Pereira, B.S.E thesis, Princeton University, Princeton, NJ, 1995.

${ }^{9}$ S. Zhu, W. G. Miller, L. E. Scriven, and H. T. Davis, "Superspreading of water-silicone surfactant on hydrophobic surfaces,' Colloids Surf., A 90, 63 (1994).

${ }^{10}$ B. Frank and S. Garoff, "Origins of the complex motion of advancing surfactant solutions,'” Langmuir 11, 87 (1995).

${ }^{11} \mathrm{~S}$. He and J. Ketterson, "Surfactant driven spreading of a liquid on a vertical surface,'” Phys. Fluids A 7, 2640 (1995). 
${ }^{12} \mathrm{~S}$. Bardon et al., "Structure and dynamics of liquid films on solid surfaces," Faraday Discuss. 104, 307 (1996).

${ }^{13}$ G. M. Homsy, "Viscous fingering in porous media," Annu. Rev. Fluid Mech. 19, 271 (1987).

${ }^{14}$ S. M. Troian, E. Herbohlzheimer, and S. A. Safran, "Model for the fingering instability of spreading surfactant drops,'” Phys. Rev. Lett. 65, 333 (1990).

${ }^{15}$ D. Halpern and J. B. Grotberg, "Dynamics and transport of a localized soluble surfactant on a thin film,"' J. Fluid Mech. 237, 1 (1992).

${ }^{16} \mathrm{O}$. E. Jensen and J. B. Grotbert, "The spreading of heat or soluble surfactant along a thin liquid film,', Phys. Fluids A 5, 58 (1993).

${ }^{17} \mathrm{~S}$. F. Shen, "Some considerations of the laminar stability of incompressible time-dependent basic flows,' J. Aerosp. Sci. 28, 397 (1961).

${ }^{18}$ P. Drazin and W. H. Reid, Hydrodynamic Stability (Cambridge University Press, New York, 1995)

${ }^{19}$ B. F. Farrell and P. J. Ioannou, "Generalized Stability Theory, Part I: Autonomous operators,' J. Atmos. Sci. 53, 2025 (1996).

${ }^{20}$ B. F. Farrell and P. J. Ioannou, "Generalized Stability Theory. Part II: Nonautonomous operators,'” J. Atmos. Sci. 53, 2041 (1996).

${ }^{21}$ D. A. Kesler, J. Koplik, and H. Levine, "Pattern selection in fingered growth phenomena,' Adv. Phys. 37, 255 (1988).

${ }^{22}$ O. K. Matar and S. M. Troian, in Dynamics in Small Confining Systems III, edited by J. M. Drake, J. Klafter, and E. R. Kopelman (Materials Research Society, Boston, 1996), Vol. 464, p. 237.

${ }^{23}$ O. K. Matar and S. M. Troian, 'Linear stability analysis of an insoluble surfactant monolayer spreading on a thin liquid film,' Phys. Fluids A 9, 3645 (1997).

${ }^{24}$ O. K. Matar and S. M. Troian, "Spreading of surfactant monolayer on a thin liquid film: Onset and evolution of digitated structures,' Chaos 9, 141 (1999).

${ }^{25}$ O. K. Matar, Ph.D. thesis, Princeton University, 1998.

${ }^{26}$ D. R. Wilson and A. F. Jones, "The entry of a falling film into a pool and the air-entrainment problem,'’ J. Fluid Mech. 128, 219 (1983).
${ }^{27}$ A. W. Adamson, in Physical Chemistry of Surfaces (Wiley, New York, 1990), Chap. 3, pp. 87-88.

${ }^{28}$ F. F. Espinosa, "Spreading of surfactant in a pulmonary airway,' Adv. Bioeng. ASME 20, 571 (1991).

${ }^{29}$ W. M. Orr, "The stability or instability of the steady motions of a perfect liquid and of a viscous liquid. Part II. A viscous liquid,' Proc. R. Ir. Acad. sect. A Math. Astron. Phys. Sci. 27, 69 (1907).

${ }^{30}$ For most of our study, the disturbances were applied at the origin of time, $\tau_{0}=1$. The definitions described, however, can equally well be used to study the impact of disturbances applied at later times $\tau_{0}$, either on an evolving base state or one frozen in time.

${ }^{31}$ W. E. Schiesser, The Numerical Method of Lines (Academic, San Diego, 1991).

${ }^{32}$ A. C. Hindmarsh, in Scientific Computing, edited by R. S. Stepleman (North-Holland, Amsterdam, 1983), p. 55.

${ }^{33}$ O. K. Matar and S. M. Troian, "Growth of nonmodal transient structures during the spreading of surfactant coated films,' Phys. Fluids A 10, 1234 (1998).

${ }^{34}$ D. P. Gaver and J. B. Grotberg, "Droplet spreading on a thin viscous film,'” J. Fluid Mech. 235, 399 (1992).

${ }^{35}$ K. M. Butler and B. F. Farrell, 'Optimal perturbations and streak spacing in wall-bounded turbulent shear flow,', Phys. Fluids A 5, 774 (1993).

${ }^{36}$ A. L. Bertozzi and M. P. Brenner, "'Linear stability analysis and transient growth in driven contact lines,'” Phys. Fluids A 9, 530 (1997).

${ }^{37}$ H. E. Huppert, "Flow and instability of a viscous current down a slope," Nature (London) 300, 427 (1982)

${ }^{38}$ S. M. Troian, E. Herbolzheimer, S. A. Safran, and J. F. Joanny, “'Fingering instabilities of driven spreading films,' Europhys. Lett. 10, 25 (1989).

${ }^{39}$ M. A. Spaid and G. M. Homsy, "Stability of Newtonian and viscoelastic dynamic contact lines,' Phys. Fluids A 8, 460 (1996).

${ }^{40}$ D. E. Kataoka and S. M. Troian, "The transient dynamics and structure of optimal excitations in thermally driven films,', Phys. Fluids (submitted). 